\title{
Photoinduced Electron Injection in a Fully Solvated Dye-Sensitized Photoanode: A Dynamical Semiempirical Study
}

\author{
Jan Paul Menzel,* Anastasios Papadopoulos, Jelena Belić, Huub J. M. de Groot, Lucas Visscher, \\ and Francesco Buda*
}

Cite This: J. Phys. Chem. C 2020, 124, 27965-27976

Read Online

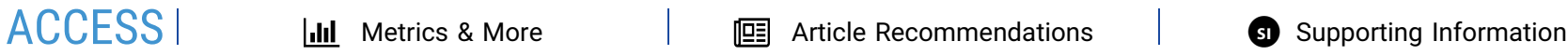

ABSTRACT: Dye-sensitized solar cells and dye-sensitized photoelectrochemical cells have attracted much interest in recent years for solar energy conversion. More effort is still required to increase the efficiency of these devices, which is closely linked to the crucial process of photoinduced charge separation. Computational studies can provide insights into this fundamental process and suggest molecular components and interfaces that feature optimal energy-level alignment before time-consuming trial-and-error experimental realization. Here, we use a combination of density functional based tight binding and an extended Hückel approach to perform quantum classical simulations of photoinduced electron injection in a $\mathrm{TiO}_{2}$ dye-

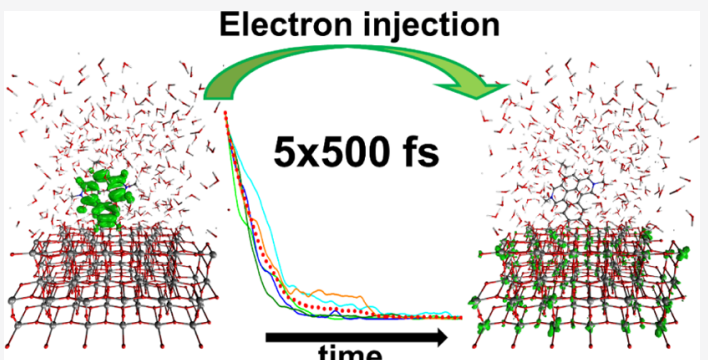
sensitized photoanode with explicit solvation at a reasonable computational cost. In particular, we evaluate injection capabilities of core-extended naphthalene diimide (NDI) dyes with three different anchoring groups. Our results stress the importance of nuclear motion as well as conformational and trajectory sampling for a realistic description of the injection process. Furthermore, explicit solvation highly influences the conformational space explored by the dye and anchoring molecules, especially concerning the adsorption mode. Taking these effects into account, the core-extended NDI with a catechol-based anchoring moiety is shown to be the most promising ultrafast electron injector. Our strategy allows for a more systematic computational search for appropriate molecular chromophores in dye-sensitized devices for solar energy conversion.

\section{INTRODUCTION}

In the search for environmentally friendly, renewable energy sources, dye-sensitized solar cells (DSSCs) have a high potential as a cost-effective alternative to silicon-based photovoltaic cells in solar energy conversion. ${ }^{1-3}$ These devices consist of semiconductor-based electrodes, which have been sensitized with photoactive molecular chromophores that absorb in the visible light range. DSSCs can also be extended by including molecular catalysts for direct conversion of solar energy to chemical fuel (dye-sensitized photoelectrochemical cells or DS-PECs). Both of these devices have attracted continued scientific interest. ${ }^{4-8}$

The anode and/or the cathode can be dye-sensitized to activate redox processes in the two half-cells. In this work, we will focus on the design of a dye-sensitized photoanode, with a schematic setup shown in Figure 1. A semiconductor electrode (here $\mathrm{TiO}_{2}$ ) is sensitized with a molecular dye [here a coreextended naphthalene diimide (NDI)]. The dye is attached via an anchoring group (here catechol). Upon photoexcitation with visible light, the excited electron is injected from the dye's lowest unoccupied molecular orbital (LUMO) into the conduction band of the electrode. This electron is then transferred via an external circuit toward the cathode. Electron flow from the cathode toward the anode via the electrolyte is then carried by redox mediators, which pick up the electron,

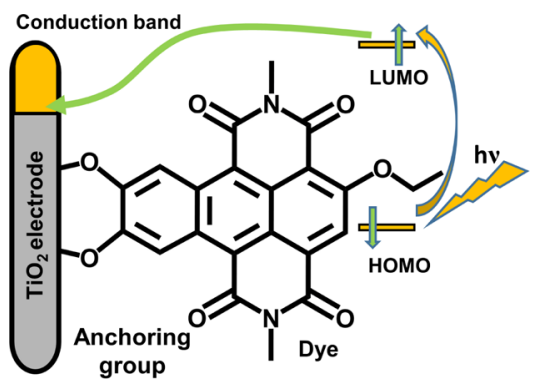

Figure 1. Characteristic setup of a dye-sensitized photoanode: a dye molecule is attached via an anchoring group to a semiconductor electrode. Upon photoexcitation to the LUMO, the dye injects an electron into the conduction band of the semiconductor.

diffuse toward the anode, and reduce the photo-oxidized dye, completing the cycle. In the case of DS-PECs, the photooxidized dye is regenerated by electrons extracted from water

Received: October 22, 2020

Revised: November 30, 2020

Published: December 9, 2020 
splitting via a suitable water oxidation catalyst, generating oxygen and protons in the process. The protons diffuse via a proton-exchange membrane toward the cathode, where, in combination with the electron, the chemical fuel is produced, for example, hydrogen from proton reduction or carbon-based fuel from $\mathrm{CO}_{2}$ reduction. ${ }^{9}$

For both types of devices (DSSCs and DS-PECs), the energetic alignment of the involved electronic states is absolutely crucial for obtaining a high forward reaction yield. The thermodynamic driving force and the electronic coupling between molecular excited state and semiconductor conduction band need to be tailored for rapid electron injection from the dye into the electrode while suppressing wasteful back-transfer and charge recombination. ${ }^{10,11}$ Finding a good compromise between these two contrasting but related requirements remains challenging.

Computational chemistry can provide insights into specific fundamental processes involved, such as interfacial electrontransfer and charge separation. ${ }^{12,13}$ It can deliver tools to screen through potential molecular dyes and interfaces to propose candidates before time-consuming synthesis and tedious experimental investigations. ${ }^{14}$ Density functional theory (DFT) based methods are often used to compare the orbital energies of the molecular dyes with the electrodes' conduction band states and localization of the involved frontier orbitals. ${ }^{15-18}$ Unfortunately, limitations of standard exchange correlation functionals may hinder the comparison with experimental values. For isolated molecular dyes, linear response time-dependent DFT (TDDFT) gives much more reliable results. ${ }^{19-21}$

However, these static approaches cannot capture changes in the electronic wave functions as the molecular conformations change. In addition, delocalization and localization effects, as well as changing the orbital character due to, for example, forming or breaking of conjugation, are not described either. These effects can be included in a limited way by ensemble averaging, taking geometries from equilibrated molecular dynamics (MD) runs. However, changes in the geometry during the injection process itself remain elusive. Finally, interaction of different electronic states can turn out to provide a pathway dominating the injection process when the electronic system is propagated.

Real-time evolution of the photoexcited electron can provide important insights into the dynamic aspect of these ultrafast injection processes. Nonadiabatic MD (NAMD) approaches would be a favorable method because they include the coupling between electronic and nuclear dynamics, but in practice, this is still very expensive to use in these extensive systems over long time scales. ${ }^{2-26}$ Both surface hopping and Ehrenfest-type approaches based on (TD-)DFT have been successfully used to simulate electron injection processes. ${ }^{27-31}$ These methods are computationally demanding and thus strongly limited with respect to the size of the system that can be investigated, especially if one is interested in performing several trajectories and compare numerous dye semiconductor systems and their respective performances.

An alternative, a computationally very efficient route to simulate the electron injection from photoexcited dye molecules, was put forward by Rego and Batista. ${ }^{32}$ In this method, the photoinduced electron dynamics is modeled via quantum dynamics based on an extended Hückel Hamiltonian. The approach was first demonstrated with a simulation of photoinduced electron injection from catechol into a $\mathrm{TiO}_{2}$ anode. ${ }^{32}$ It has been successfully used to elucidate electrontransfer processes in a range of systems, including $\mathrm{TiO}_{2}$-based electrodes with organic dyes, ${ }^{32-38}$ DSSCs with transition metal complex-based sensitizers, ${ }^{39-46}$ nanocrystals, ${ }^{47,48}$ and charge separation dynamics in metal complexes. ${ }^{49,50}$ While in earlier simulations using this approach the electron dynamics were done on fixed nuclear geometries, later implementations included the use of precalculated nuclear trajectories to model the effect of nuclear dynamics on the electron injection process. $^{37,49,51}$ Within this efficient, semiclassical approach, switching between the adiabatic molecular orbital (MO) basis and the diabatic atomic orbital ( $\mathrm{AO}$ ) basis allows for nonadiabatic population transfer. More recently, this method has also been extended to use the nonadiabatic elements derived from the quantum mechanically treated electronic system as an additional force in the classical MD to allow for persistent change in the nuclear trajectory as a response to the evolution of the electronic subsystem. ${ }^{52-55}$

Here, we investigate the electron injection properties of an extended core NDI dye with different anchoring groups in a $\mathrm{TiO}_{2}$ based-dye sensitized photoanode using a combination of self-consistent charge density functional based tight binding (SCC-DFTB) for the nuclear dynamics and the beforementioned extended Hückel method for the electron-transfer dynamics (ETD). The main goal is to give an overview of the relative importance of nuclear motion, influence of different initial conformations and trajectories, as well as inclusion of explicit solvation. In particular, explicit solvation can have several different effects on the photoinduced electron injection process: (i) the solvent can change the absorption properties of the molecular dye, (ii) it has an influence on the configurational space explored specifically at the dye-electrode interface, (iii) water tends to coadsorb onto the semiconductor interface and polarize the surface, thereby changing the band structure and energy of the surface states, and (iv) the water can interact more directly with the anchored dye via breaking bonds between the anchor and electrode, or by forming hydrogen bonds.

We aim to determine the most appropriate anchoring group for our dye with respect to their electronic conductivity and injection properties. For this, three benzene-derived anchoring moieties were chosen: benzoic acid, catechol, and benzohydroxamic acid. Carboxylic acids are probably the most widely used anchoring groups but lack chemical stability, whereas catechol and benzohydroxamic acid show reasonable chemical stability and high estimated electron injection capabilities. ${ }^{56}$ The investigated anchoring molecules are shown in Figure $2 a-$ c.

As a molecular photosensitizer, an NDI-based dye was used, as NDIs are powerful light absorbers. They have a high extinction coefficient and can be tuned over the entire visible light range by introduction of electron-withdrawing or -donating groups. ${ }^{14,57,58}$ In Figure $2 d-f$, the investigated core-extended ethoxy NDIs, with the anchoring moieties fused into the extended core, are shown, which will be named cat-NDI, ben-NDI, and hyd-NDI, according to their respective anchoring group.

The article is organized as follows: first, a description of the computational methods is given, which includes a short overview of the general procedure, the construction of the photoanode system in silico, treatment of the nuclear subsystem, and finally the ETD. In the following section, we discuss our results, with special focus on the effect of nuclear 
a)<smiles>Oc1ccccc1[Hg]</smiles>

b)<smiles>O=C(O)c1ccccc1</smiles>

c)

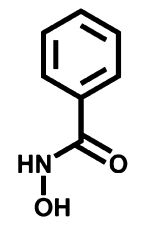

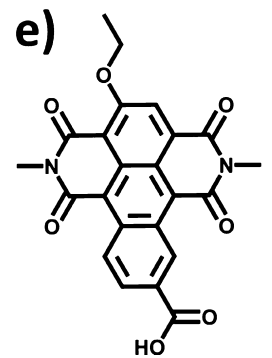

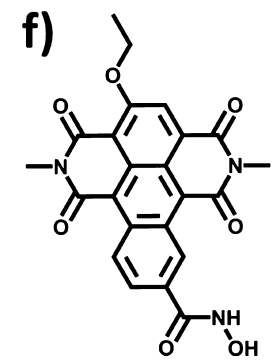

Figure 2. Investigated anchoring and dye molecules: (a) catechol, (b) benzoic acid, (c) benzohydroxamic acid, (d) core-extended ethoxyNDI with a catechol anchoring group (cat-NDI), (e) core-extended ethoxy-NDI with carboxylic acid anchoring group (ben-NDI), and (f) core-extended ethoxy-NDI with hydroxamic acid anchoring group (hyd-NDI).

dynamics, trajectory averaging, and explicit solvation. Conclusions are given in the last section.

\section{COMPUTATIONAL METHODS}

General Procedure. To evaluate the performance of the chosen dye and anchoring molecules, a quantum classical semiempirical method is used, which allows for simulations of the full photoanode system at a relatively low computational cost. Interfacial electron-transfer simulations based on quantum dynamics using the $\mathrm{AO} / \mathrm{MO}$ propagator and an extended Hückel Hamiltonian ${ }^{50}$ are performed on a priori generated nuclear trajectories obtained at the SCC-DFTBMD level. ${ }^{59-61}$ The extended Hückel Hamiltonian is parameterized on DFT results (see SI-3 for detailed information). The general workflow is described in Scheme 1 .

The DFTB module of the Amsterdam Density Functional (ADF) program was used for simulating the nuclear

Scheme 1. Preparation of Nuclear Trajectories and Extended Hückel Parameters Used in the Semiempirical Quantum Classical Simulations of Photoinduced Electron Injection $^{a}$

\section{Workflow}

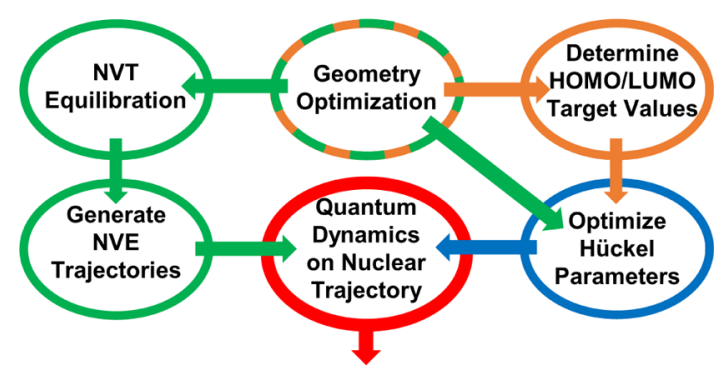

Electron injection

${ }^{a}$ Colors refer to different computational methods: green for SCCDFTB, orange for DFT/B3LYP, blue for Hückel parameter optimization, and red for the $\mathrm{AO} / \mathrm{MO}$ propagation using the extended Hückel Hamiltonian.

dynamics. ${ }^{62,63}$ The tiorg-0-1 parameter set, which is optimized to describe bulk $\mathrm{Ti}$ and $\mathrm{TiO}_{2}$ as well as $\mathrm{TiO}_{2}$ surfaces with and without organic adsorbants, was chosen for the SCC-DFTB. ${ }^{64}$ The ADF program was also used for determining the DFT target values for the parameter optimization. The ETD simulations were performed using the code developed by Rego and Batista. ${ }^{32}$

Constructing the Photoanode. $\mathrm{TiO}_{2}$ was assumed to be in the anatase phase because this phase is photocatalytically more active than rutile $\mathrm{TiO}_{2}$ and most often used for photoelectrodes. $^{65}$ The anatase (101) surface was created from an optimized bulk supercell containing 48 atoms and including a lattice vector optimization (see Figure S1 in the SI). A supercell of three layers for a total of 144 atoms and cell dimensions of $10.045 \AA \times 15.234 \AA \times 70 \AA$ with the surface normal to the $z$-direction was used. $\mathrm{TiO}_{2}$ was reoptimized to model surface relaxation effects, while the lowest layer was kept frozen in bulk geometry. All anchoring groups were attached via the bidentate bridging mode. The dissociating protons were adsorbed on the anatase surface to keep the unit cell overall neutral. For benzohydroxamic acid, the protonation state 1 was chosen (one proton is dissociated, one remains) as determined by Rudshteyn et al. ${ }^{66}$ The optimized geometries of these anatase-anchor molecule complexes are shown in Figure S2a$c$ in the SI.

Water molecules were added to obtain a total density of $1 \mathrm{~g} /$ $\mathrm{mL}$. Figure $3 \mathrm{a}$ shows this simulation box specifically for

\section{a)}

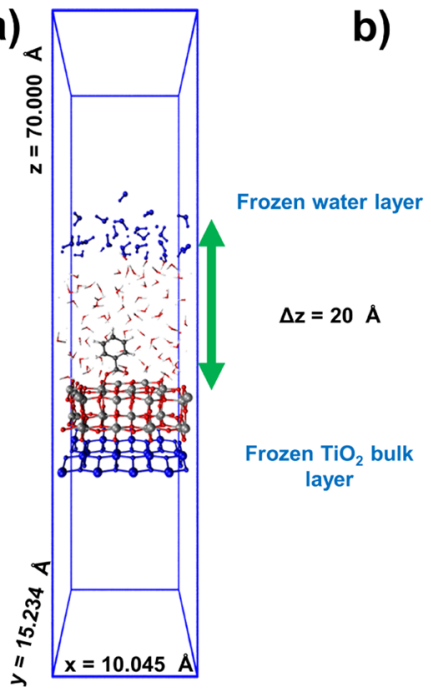

Figure 3. Simulation boxes for (a) benzoic acid and (b) ben-NDI attached to a $\mathrm{TiO}_{2}$-slab including explicit solvation. Periodic boundary conditions are enforced in $x, y$, and $z$. Shown in blue are atoms kept frozen to mimic the $\mathrm{TiO}_{2}$ bulk geometry and to prevent evaporation and diffusion of the water molecules toward the opposite side of the $\mathrm{TiO}_{2}$ slab.

benzoic acid, containing a total of 453 atoms. SCC-DFTBbased $\mathrm{MD}$ simulations were performed while freezing the lowest $\mathrm{TiO}_{2}$ layer to maintain the bulk geometry, as well as a layer of water on top to prevent evaporation. The simulation box for ben-NDI, including a total of 1329 atoms and box dimensions of $20.091 \AA \times 22.851 \AA \times 70 \AA$, is shown in Figure 3b.

Generating Nuclear Trajectories. In Scheme 2, the general procedure to generate the nuclear trajectories is shown. First, a $\mathrm{MD}$ equilibration run in the NVT ensemble, using a 
Scheme 2. Geometry and Trajectory Generation for Usage in the ETD Simulations ${ }^{a}$

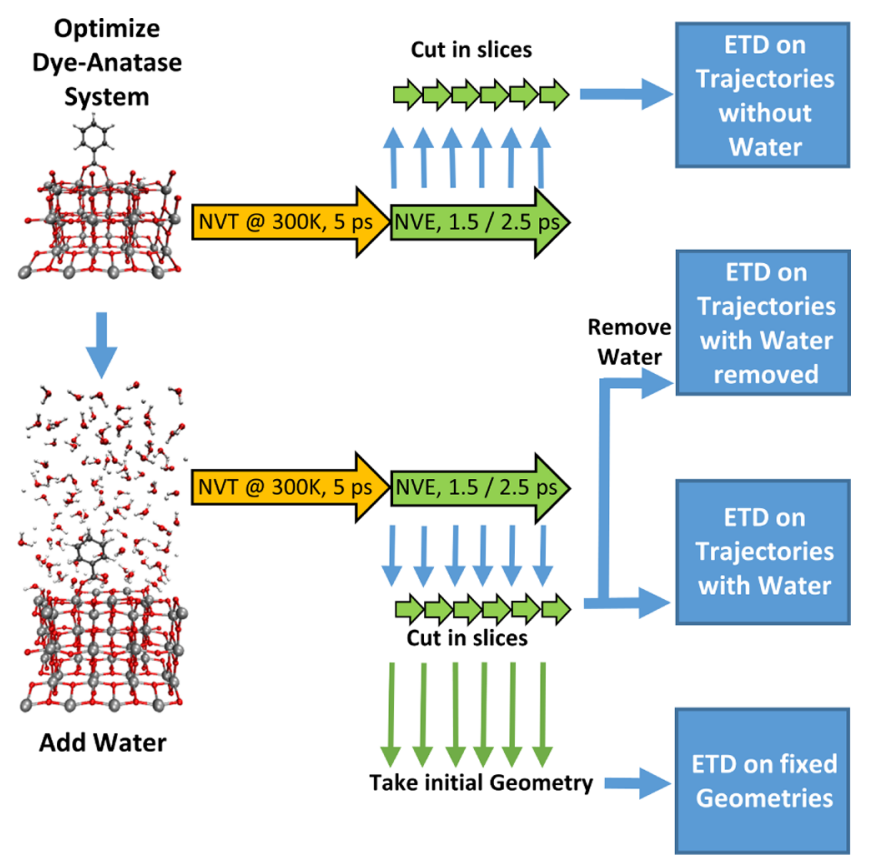

${ }^{a}$ After an initial NVT equilibration at $300 \mathrm{~K}$ (yellow arrow), an NVE production run is performed (green arrow). This trajectory is then split into different slices for the quantum propagation. This procedure is performed twice, with and without explicit solvation. ETD simulations on static nuclear geometries were performed on the initial geometries of the trajectory slices.

Berendsen thermostat ${ }^{67}$ at $300 \mathrm{~K}$, was performed for 5 ps with a time step of $1 \mathrm{fs}$. This was followed by a total of $1.5 \mathrm{ps}$ in the NVE ensemble for the anchoring groups, while 2.5 ps was produced for the core-extended NDI dyes. A time step of $0.1 \mathrm{fs}$ was used for both. This short time step is necessary for stable quantum dynamics simulations. The resulting trajectory was then split into 15 parts of $100 \mathrm{fs}$ each in the case of the anchoring groups. For the dyes, five slices of 500 fs each were taken. The initial geometries of these shorter trajectories were used for the ETD simulations on a fixed geometry.

To estimate the influence of explicit solvation, three different cases were investigated: full explicit solvation during the nuclear trajectory generation and the ETD, the same nuclear trajectories but removing the water molecules for the ETD, and nuclear trajectories and ETD without any water molecules. In this way, we can disentangle pure polarization effects because of the solvent from changes in the conformations explored during the dynamics, especially associated with the formation of hydrogen bonds and changes of adsorption mode.

Electron-Transfer Dynamics. The ETD simulations were performed using an extended Hückel Hamiltonian that is based on parameterization of Coulomb and overlap integrals. For the water molecules and anatase electrode, the standard Hückel parameters were used. ${ }^{68}$ The Hückel parameters for the dye and anchor molecules were optimized to achieve good relative energy alignment of the conduction band edge of the anatase on the one hand and the frontier orbitals of the organic molecule on the other hand as determined by experimental values and DFT results (see Section S3 in the SI). Consistent with the interpretation of the eigenvalues of the Hückel Hamiltonian as single particle energies, Hückel parameters were optimized on the dye and anchoring molecules in implicit water using $\triangle$ SCF-derived values for the highest occupied molecular orbital (HOMO) and $\triangle$ SCF plus excitation energy (TDDFT or experimental) for the LUMO energies at the DFT-B3LYP ${ }^{69,70} / \mathrm{DZP}^{71}$ level with implicit COSMO ${ }^{72}$ water. A more detailed description and comparison to available experimental values is given in Section S3 in the SI.

The excitation of each organic molecule is modeled by localizing the photoexcited electron on the LUMO of the corresponding adsorbed molecule, with the hole being localized at its HOMO. Even though there is evidence that catechol adsorbed to an anatase surface is excited directly to a charge-transfer state with the electron already in the conduction band of $\mathrm{TiO}_{2}{ }^{73}$ the local excitonic state is used here because we are interested in the ability of catechol to inject a photoexcited electron donated by an excited dye. During the time evolution of these wave packets, the electron and hole population were calculated for the following fragments: the anatase electrode, the adsorbed organic molecule, and the water molecules.

\section{RESULTS AND DISCUSSION}

Nuclear Dynamics. During the equilibration dynamics with the explicit solvent, water adsorption onto the anatase surface is observed in all systems considered in this work. Therefore, the initial hydrogen bond formation toward the oxygen located on the surface steps (Figure 4a) is followed by

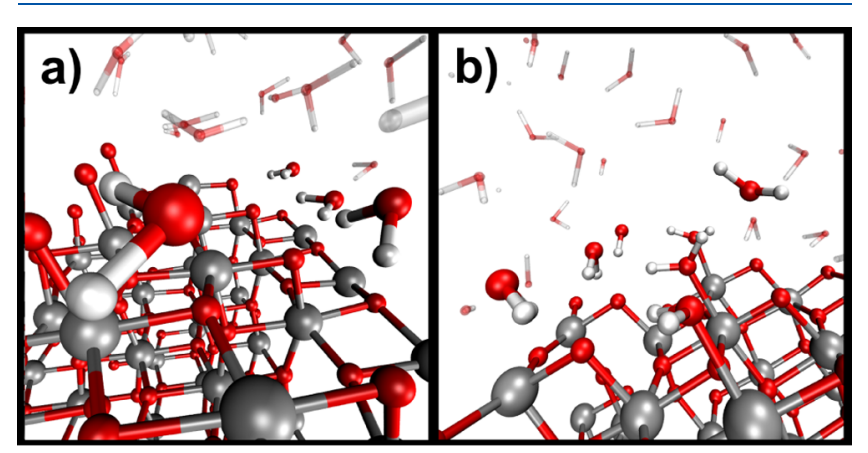

Figure 4. Adsorption of water on the anatase (101) surface: (a) initially, water molecules associate via hydrogen bonds with the oxygen on the surface step; the corresponding adsorbed water molecules are shown in bold. (b) Covalent bonds to $\mathrm{Ti}$ atoms are formed and hydrogen bonds to the bulk water create further water layers that remain relatively dynamic.

covalent attachment of the water oxygen to titanium (Figure 4b). After adsorption, hydrogen bond formation to the surface oxygen, coadsorbed water molecules, and bulk water is also common (see also Figure 4b). These findings are consistent with the work by Selli et al. and Aschauer et al. that found these geometries for multilayers of water on anatase $(101) .^{74,75}$

Interestingly, the adsorption mode of benzohydroxamic acid changes during the $\mathrm{MD}$. While the bidentate adsorption is most stable in vacuum, with explicit water, it switches spontaneously to a monodentate mode with hydrogen bond formation to a coadsorbed water molecule. This happens via a water molecule in solution that attacks the oxygen-titanium bond by forming a hydrogen bond to the hydroxamic acid oxygen (Figure 5a). Afterward, the molecule rearranges to form a hydrogen bond to a neighboring adsorbed water molecule (see Figure 5b). This mode was also found to be the 


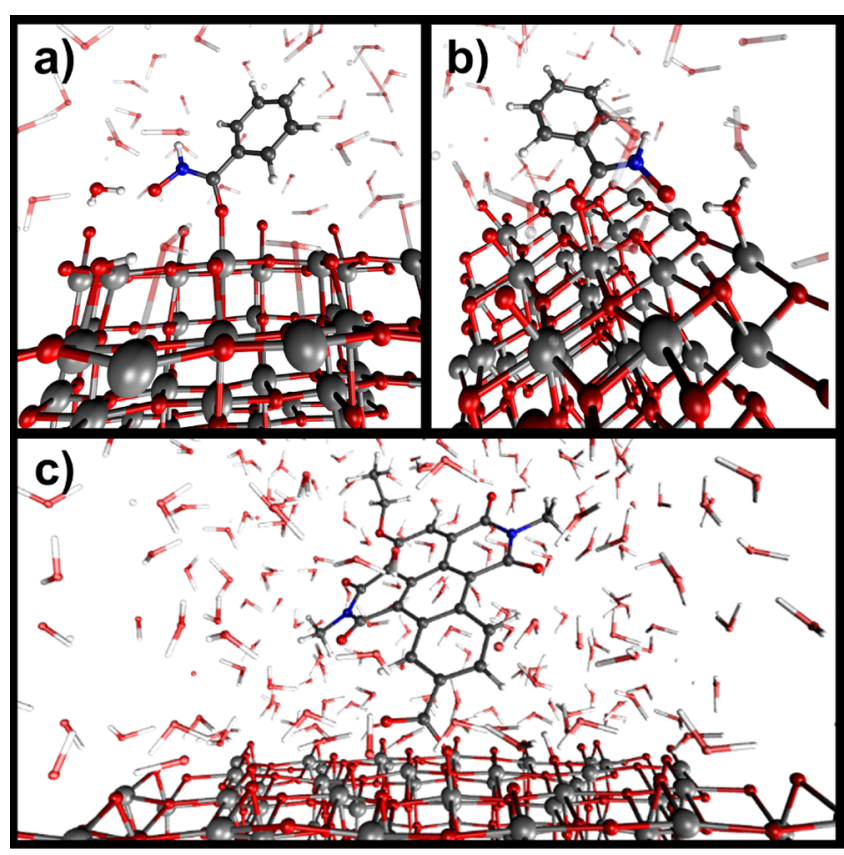

Figure 5. Change of benzohydroxamic acid adsorption mode from bidentate to monodentate via (a) attack of a water molecule (highlighted) and (b) rearrangement forming a hydrogen bond to the highlighted coadsorbed water molecule, and (c) monodentate adsorption of ben-NDI due to steric stress induced by the imide part of the NDI pointing toward the surface.

most stable with monolayer water present by Rudshteyn et al. ${ }^{66}$ It is noteworthy that SCC-DFTB-based MD is able to predict this correctly.

This change in adsorption mode from monodentate to bidentate is also present for ben-NDI and hyd-NDI. However, it also happens without explicit water, contrary to benzohydroxamic acid. This change seems to be due to the steric stress of the imide part of the NDI that points toward the surface. This leads to angular strain on the anchoring group, which is released by breaking one of the bonds to the surface. An example is shown in Figure 5c. In the case without explicit water, the attractive interaction between the $\pi$-system and the anatase surface also plays a role, while the adsorbed water prevents this interaction when including explicit solvation. In the hyd-NDI case, this leads to $\pi$-stacking between the entire aromatic plane of the NDI and the surface.

Electron Injection Properties of Anchoring Molecules. The electron injection over time for one ETD simulation on a single trajectory with explicit solvation is given in Figure 6a for benzohydroxamic acid, where we show the electron population on different fragments over time. At the beginning of the simulation, both the hole and the electron are completely localized on benzohydroxamic acid, occupying the respective HOMO and LUMO orbitals (see Figure 6c). During the quantum dynamics evolution, the photoexcited electron is transferred from benzohydroxamic acid to the anatase electrode. As can be seen, electron injection is complete within $20 \mathrm{fs}$ (see Figure 6a). No electron density is donated to the explicit water molecules. The hole population (see Figure 6b) remains stable on benzohydroxamic acid. At the end of the simulation, the electron is therefore injected into the electrode, while the hole remains on benzohydroxamic acid: charge separation has occurred (see Figure 6d).

However, one single trajectory can be misleading in drawing conclusions on the injection process. To obtain a reasonable estimate of the electron injection rate, several ETD results have to be averaged. In Figure $7 a$, the photoinduced electron injection from benzohydroxamic acid to the anatase electrode is averaged over all 15 ETD simulations performed on nuclear trajectories with explicit water. The same is shown for the hole in Figure $7 b$.

Photoinduced electron injection is observed; however, the average rate is significantly lower than for the single trajectory discussed earlier: within about $60 \mathrm{fs}$ on average, the injection is basically complete. This highlights the importance of appropriate statistics by averaging over multiple simulations in determining the injection rate.

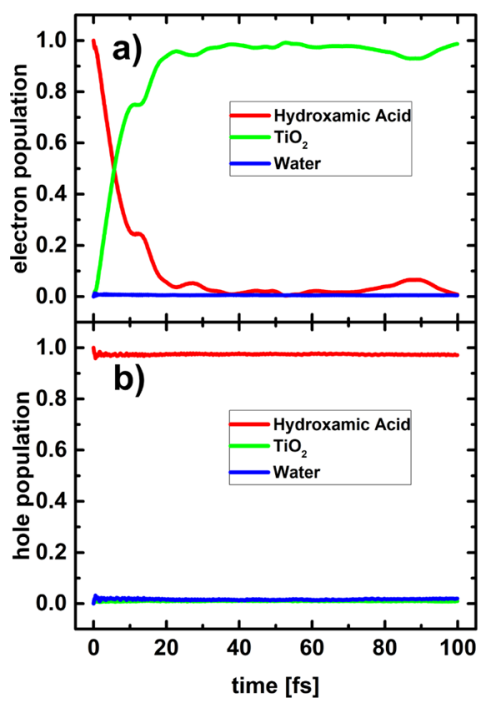

c)

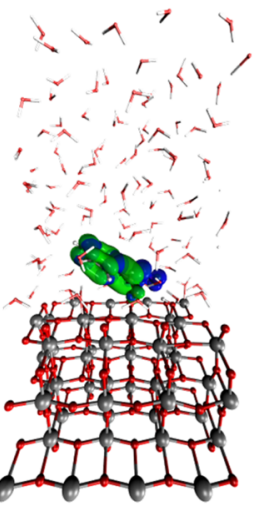

0 fs d)

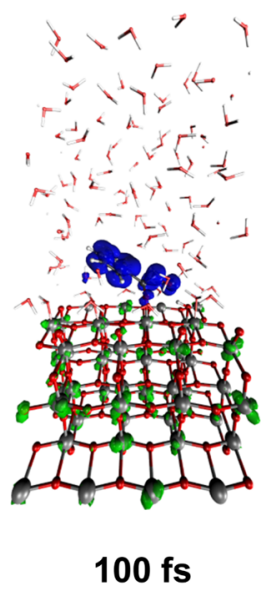

Figure 6. Electron (a) and hole (b) populations on the different fragments: benzohydroxamic acid (red), $\mathrm{TiO}_{2}$ (green) and water (blue) with full explicit solvation, (c) electron (green) and hole (blue) density at the beginning of the simulation, both fully localized on benzohydroxamic acid. (d) Electron (green) and hole (blue) density after 100 fs. The electron is injected into the $\mathrm{TiO}_{2}$ electrode, while the hole remains stable on benzohydroxamic acid. 


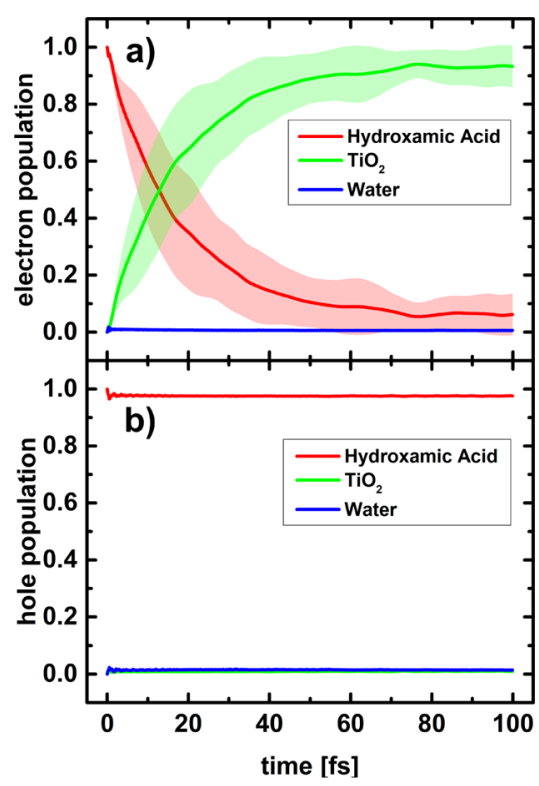

Figure 7. Electron (a) and hole (b) populations on the different fragments: benzohydroxamic acid (red), $\mathrm{TiO}_{2}$ (green), and water (blue) with full explicit solvation, averaged over 15 trajectories. The lines denote the mean values, and the shaded area denotes the standard deviation centered on the mean value.

Some general statements can be made for all simulations and for all investigated molecules: (i) no significant electron population is observed on the water molecules and (ii) the hole remains stable on the organic molecule. Figures of electron and hole survival for all cases and molecules can be found in the Supporting Information.

The importance of averaging over several geometries or trajectories is evident in Figure $8 \mathrm{a}-\mathrm{d}$, where we report the electron injection evolution for each of the 15 geometries (Figure 8a) and trajectories (Figure $8 \mathrm{c}$ ) of benzohydroxamic acid in explicit water, with the mean value and the standard deviation also included (Figure $8 \mathrm{~b}, \mathrm{~d}$ ). Figure $8 \mathrm{a}$ shows that the choice of the initial geometry can lead to qualitatively different results: from almost no injection to a very fast process finished within 20 fs. Correspondingly, this leads to a high standard deviation and a slow, incomplete injection when considering the mean value, shown in Figure $8 \mathrm{~b}$. We have analyzed in some more detail these different conformations to search for characteristic geometrical features that might affect the injection properties. While some features, such as a large dihedral angle between phenyl group and hydroxamic acid, as well as a large distance between anchoring group and $\mathrm{TiO}_{2}$ often slow down the injection process, more complex collective geometric variables seem to be involved, not only including the chromophore but also the solvent and the semiconductor surface. It is therefore challenging to disentangle these features.

The effect of including dynamics is shown in Figure 8c. Here, more geometries are explored during the trajectory and the rates change significantly compared to the static case. This means that if the injection is hindered at a certain geometry, then the injection might be slowed down in the dynamic case, but not completely inhibited. For example, the value of the dihedral angle between the anchoring group and the aromatic system can prevent electron flow from the molecule to the anatase surface, but when a more favorable conformation is explored during the trajectory, the injection channel is opened (see a more detailed description in SI-4). In previous investigations of photoinduced electron-transfer processes, coherent effects between nuclear and electronic motion have been shown to be relevant in facilitating the charge separation. ${ }^{24,26,37,52}$ Here, we have also explored possible resonances between electronic and nuclear frequencies and show an example in Section S-5 in the Supporting Information. This suggests that the electronic system is able to take advantage of nuclear modes of appropriate frequency.

A further effect of the nuclear dynamics is the larger conformational sampling within the simulation, leading to a

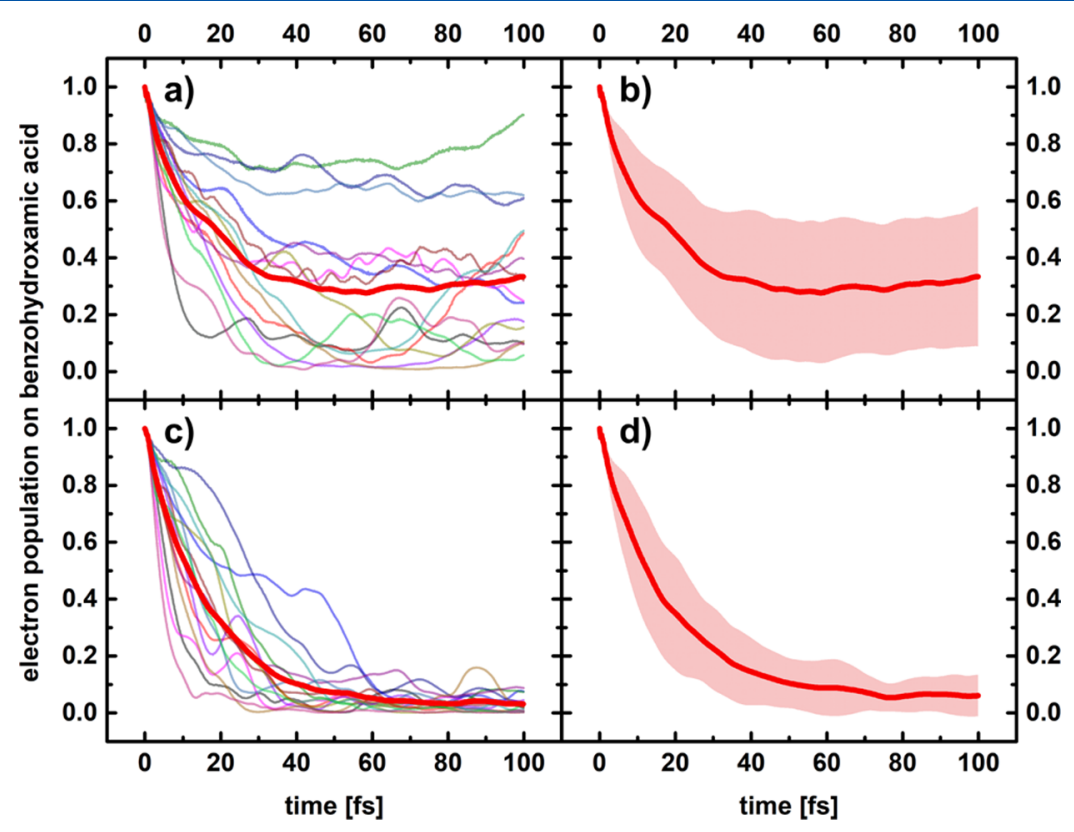

Figure 8. Variation of electron population on benzohydroxamic acid: (a) all 15 ETDs on static geometries with the mean value in bold, (b) the corresponding standard deviation (shaded area) centered on the mean value (bold line), (c) all the 15 ETDs on nuclear trajectories with the mean value in bold, and (d) the corresponding standard deviation (shaded area) centered on the mean value (bold line). 
significant decrease in the standard deviation (see Figure $8 \mathrm{~d}$ ). Benzohydroxamic acid is the most extreme case; for benzoic acid and catechol, the variation between single ETD runs is significantly lower (see Sections SI-6-SI-8).

The lower injection rate and large standard deviation of the static case is a consequence of the high variation between single trajectories and charge oscillations between the surface and molecule visible in Figure 8a. These oscillations are an effect of the finite size of the system that has been overcome in the past by including absorbing potentials in the lowest $\mathrm{TiO}_{2}$ layer. ${ }^{32,34,46}$ However, the oscillations are greatly reduced when including nuclear dynamics (see Figure 8c) because the dynamic disorder rapidly localizes the charge within the electrode. This points to a sufficient size of our system without the need of using an artificial absorbing potential, as localized low energetic states are available through thermal noise in the $\mathrm{TiO}_{2}$ slab. Because this is a dynamic effect within the electrode, the $\mathrm{TiO}_{2}$ slab needs to be treated dynamically, as freezing the surface leads to the same oscillatory behavior observed in the completely static system (see Figure S12 in the SI).

To check the relative importance of nuclear dynamics, trajectory averaging, and explicit solvation on the electron injection process, we compare the average electron population over time on all three anchoring molecules for these different cases in Figure 9. In all panels, only the electron population averaged over all 15 ETDs is shown. Single runs and standard deviations are omitted for clarity and reported in the Supporting Information (SI-6-SI-8).

In Figure 9a, the average electron population over time on all three anchoring molecules is given for the static versus dynamic case. It is important to note that the geometries chosen are the initial structures from the trajectories (see Scheme 2), indicating that the initial conditions for the static and dynamic cases are identical. The importance of nuclear dynamics for the description of the injection process is clearly visible. The electron injection is slower and less complete for all static cases in comparison to the dynamic ones because of larger differences in rate between single ETD runs as well as oscillations between the anchoring molecule and the electrode. However, this effect varies by the anchoring molecule. While for benzoic acid, especially the initial injection seems quite well described on static geometries, the difference between static geometries and nuclear trajectories is quite significant for catechol and very large for benzohydroxamic acid. All in all, nuclear dynamics are quite important in the electron injection process. More conformations are explored during the trajectory, leading to smaller differences between single ETD runs. Conformations that inhibit electron injection do not prevent the process; as over time, other parts of conformational space are explored that allow for electron flow. Furthermore, the dynamic disorder inhibits oscillations between the different fragments.

The importance of including explicit solvation is less straightforward. As mentioned earlier, to disentangle pure polarization effects from the influence of water on the conformational space explored, we investigated three cases: (i) nuclear trajectories obtained with full explicit solvation, (ii) the same trajectories but with the water molecules subsequently removed, thus keeping the effect on nuclear dynamics but removing polarization effects, and (iii) trajectories obtained in vacuum.

We first consider the pure polarization effect by comparing the ETDs of the full explicit solvation model and the same

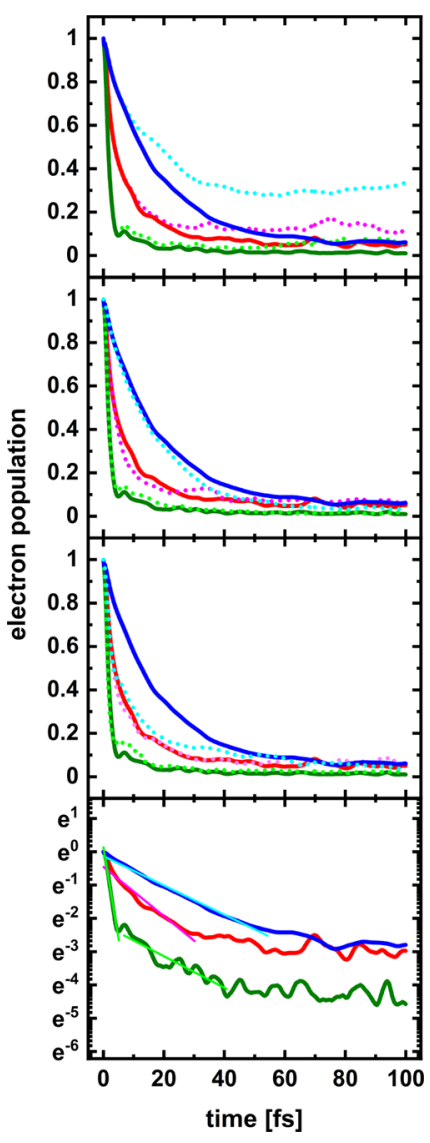

Figure 9. Electron population on catechol (red), benzoic acid (green), and benzohydroxamic acid (blue) averaged over 15 ETD simulations each. In different panels, we compare the results obtained with the nuclear trajectories with explicit water (full lines) with the results obtained with (a) static nuclear geometries (dotted lines), (b) the same nuclear trajectories with the water molecules subsequently removed for the ETD (dotted lines), and (c) trajectories obtained without explicit solvation (dotted lines). Panel (d) shows the electron injection for the fully solvated, dynamic case on a ln scale including a linear fit indicated in a lighter color.

nuclear trajectories with the water molecules removed for the quantum dynamics, as given in Figure 9b. For all the three anchoring molecules, the difference between full explicit solvation and removing the water molecules for the ETD is quite small. The presence of water slightly slows down the injection into the $\mathrm{TiO}_{2}$ electrode for benzohydroxamic acid, while benzoic acid remains almost unchanged. For catechol, injection without water is slightly increased at the beginning, but a small peak at 35 fs denotes a small back transfer that is suppressed when including explicit water. The differences decrease over the course of the simulation. We expect that the polarization effect is so small because of the tight binding character of the Hückel method, which poorly describes longrange effects.

While the polarization effect of water in our simulations is small, the effect of excluding water in the nuclear dynamics can be significant, as can be seen in Figure 9c. While the conformational space explored with water seems to be very similar for catechol, as there is almost no difference in injection rate, this is not the case for the other two anchoring molecules. There is a quite clear back-transfer peak after the initial injection (at $5 \mathrm{fs}$ ) in the case of benzoic acid that is significantly higher when excluding water. This can partially be 
explained by the missing polarization effects, as was the case in Figure $8 \mathrm{~b}$, but is more pronounced and might hint at a lower dynamic disorder in the system. In both cases, full injection is achieved after about 40 fs. For benzohydroxamic acid, the effect of explicit solvation is quite striking as the electron injection is a lot slower. This is most likely because of the changed adsorption mode discussed earlier, where benzohydroxamic acid adsorbs bidentally in vacuum but switches to monodentate adsorption mode when including explicit water (as shown in Figure 5). Explicit solvation therefore seems to be very important for benzohydroxamic acid, less so for benzoic acid, and negligible for catechol. In addition, conformational differences induced by water and direct interaction via formation of hydrogen bonds appear more important than polarization effects in this tight binding framework.

To compare the injection rates for the three anchoring molecules, we give the electron population over time on a semilogarithmic scale in Figure 9d. Here, we only focus on the fully solvated dynamic case. For all the three anchoring molecules, the injection behavior seems to follow the exponential behavior, until less than $10 \%$ electron population remains on the dye. After that, noise takes over. A linear fit is given for this part of the electron injection plot to estimate the rate. The slope, adjusted $R^{2}$ values, estimated rates, and mean life time for the three anchoring molecules are given in Table 1. Benzoic acid seems to have two distinct regimes: within the

Table 1. Slope, Adjusted $R^{2}$ Values, and Estimated Electron Injection Rates for Catechol, the Two Regimes of Benzoic Acid and Benzohydroxamic Acid Determined from the Linear Fits on the Semilogarithmic Mean Value of 15 ETD Simulations Performed on Fully Solvated Nuclear Trajectories

\begin{tabular}{lcccc}
\multicolumn{1}{c}{ molecule } & $\begin{array}{c}\text { slope } \\
{\left[\mathrm{fs}^{-1}\right]}\end{array}$ & $\begin{array}{c}\text { adjusted } \\
R^{2}\end{array}$ & $\begin{array}{c}\text { estimated electron } \\
\text { injection rate }\left[\mathrm{s}^{-1}\right]\end{array}$ & $\begin{array}{c}\text { mean life } \\
\text { time }[\mathrm{fs}]\end{array}$ \\
catechol & -0.074 & 0.95397 & $7.4 \times 10^{13}$ & 13.6 \\
benzoic acid & -0.547 & 0.98888 & $5.5 \times 10^{14}$ & 1.8 \\
& -0.048 & 0.87008 & $4.8 \times 10^{13}$ & 21.1 \\
$\begin{array}{l}\text { benzohydroxamic } \\
\text { acid }\end{array}$ & -0.043 & 0.99302 & $4.3 \times 10^{13}$ & 22.8 \\
\hline
\end{tabular}

first 5 fs, a very rapid injection, followed by a slower rate comparable to the other two anchoring groups. This is most likely a relaxation effect due to the strong coupling between $\mathrm{TiO}_{2}$ surface states and the oxygens of benzoic acid, as can be seen in the density of states given in the SI (Figure S4). The injection rates are all in the order of $10^{13} \mathrm{~s}^{-1}$, with the first regime of benzoic acid showing an even higher rate of the order of $10^{14} \mathrm{~s}^{-1}$.

All in all, conformational averaging and inclusion of nuclear dynamics are crucial in describing the exponential decay of the injection process, while explicit solvation seems important for the correct description of nuclear dynamics, but not in the ETD simulations. While the injection rates vary when including or excluding nuclear dynamics and explicit solvation, the relative trend of the anchoring molecules stays the same.

Electron Injection Properties of Core-Extended NDI Dyes with Different Anchoring Groups. The electron injection properties of the excited anchoring molecules give some insights into the effects of nuclear motion and explicit solvation on these molecules as well as an estimate of their performance. In this section, we focus on the electron injection properties of the whole photoanode system including the NDIbased dyes shown in Figure 2d-f.

A comparison of the average electron population over time on these NDI-based dyes is given in Figure 10a-d. Here, we

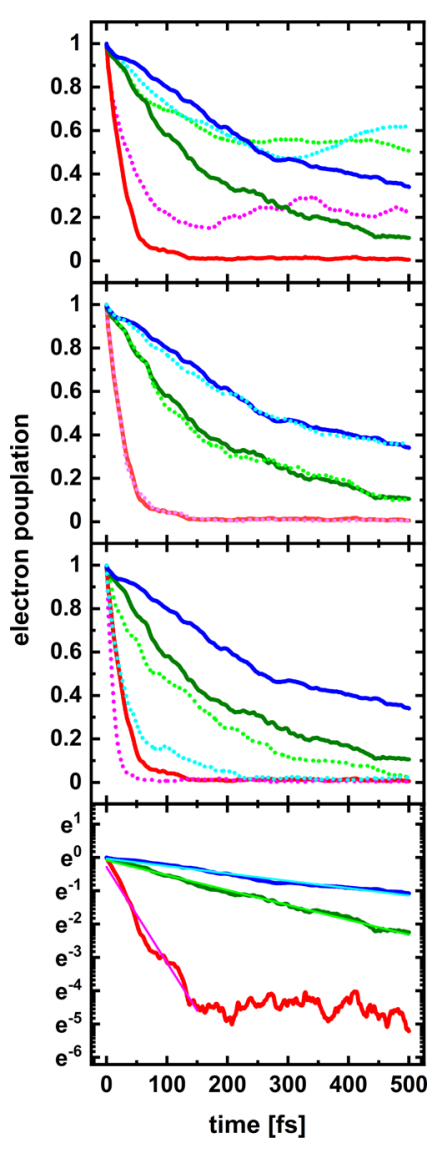

Figure 10. Electron population on cat-NDI (red), ben-NDI (green), and hyd-NDI (blue) averaged over five ETD simulations each. In different panels, we compare the results obtained with the nuclear trajectories with explicit water (full lines) with the results obtained with (a) static nuclear geometries (dotted lines), (b) the same nuclear trajectories with the water molecules subsequently removed for the ETD (dotted lines), and (c) trajectories obtained without explicit solvation (dotted lines). Panel (d) shows the electron injection for the fully solvated, dynamic case on a ln scale including a linear fit indicated in a lighter color.

focus on the time evolution of the mean values averaged over five ETD simulations of 500 fs each. Figures with single trajectories and standard deviations can be found in the SI (Section SI-9-SI-11). In Figure 10a, we compare the electron injection evolution obtained with ETDs on static geometries with those performed on nuclear trajectories. Again, the geometries correspond to the initial structures of the trajectories, indicating that initial conditions are the same. In general, the electron injection here is significantly slower in comparison to the corresponding benzene-derived anchoring molecules. This might be associated with both lower LUMO energies (and therefore lower driving force) and lower coupling of the molecules' LUMO to the conduction band states as the distance between the donor and acceptor is increased. Although cat-NDI fully injects the photoexcited electron within $150 \mathrm{fs}$ in the dynamic case, the exclusion of nuclear dynamics leads to a far lower injection with an electron 
population on the dye molecule of still $20 \%$ after $500 \mathrm{fs}$. The same holds true for ben-NDI, where the electron population left on the dye rises from $10 \%$ in the dynamic to approximately $50 \%$ in the static case. For the hyd-NDI, this is even more striking: while the electron population on the dye is about $35 \%$ after $500 \mathrm{fs}$ when including nuclear dynamics, in the static case, it actually rises from a minimum of $45 \%$ at 300 fs to about $60 \%$ after $500 \mathrm{fs}$.

Furthermore, the importance of explicit solvation in the trajectory generation is much more obvious here than for the benzene derivatives. While the removal of water molecules for the ETD leads to very little change (dotted lines in Figure 10b in comparison to the full lines), the electron injection rate is significantly higher for all dye molecules when using nuclear trajectories obtained without explicit solvation (dotted lines in Figure 10c). Interestingly, the trend of the injection properties changes also qualitatively. While in all cases, electron injection is quicker without explicit water, the hyd-NDI shows an extreme increase in injection rate and surpasses ben-NDI. This extreme change can be explained by the $\pi$-stacking of the hydNDI onto the surface that was mentioned earlier. Such an interaction leads to very strong electronic coupling between the NDI and $\mathrm{TiO}_{2}$ that allows for direct injection between the dye and surface without crossing the anchoring molecule (see Figure 11). Consequently, it is absolutely crucial to include explicit solvation for the generation of nuclear trajectories to predict the performance of a molecular dye.

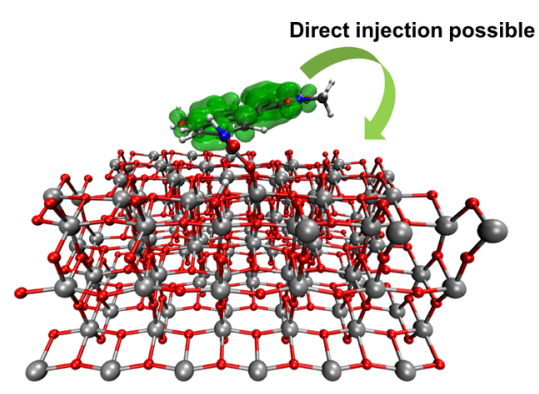

Figure 11. Representative geometry of hyd-NDI without explicit solvation. The close interaction between the $\pi$-system of the dye with the anatase surface allows for direct injection. The electron density (shown in green) does not need to pass through the anchoring moiety.

In Figure 10d, the electron population on the core-extended NDI dyes averaged over five ETDs on trajectories with explicit water is shown on a semilogarithmic scale. This allows for a linear fit to estimate the injection rates. In comparison to the anchoring groups, the exponential behavior is even more evident. Slopes, adjusted $R^{2}$ values, estimated rates, and mean survival time are given in Table 2. The rates of injection are significantly lower than for the anchoring molecules. Still, the slowest injection (the hyd-NDI) has a mean life time of about $500 \mathrm{fs}$, still $4-5$ orders shorter than a typical fluorescence life time. The most effective anchor-dye complex is thus cat-NDI, which is 1 order of magnitude faster than the other two molecules. Ben-NDI and hyd-NDI have comparable rates, with ben-NDI approximately twice as fast. Contrary to the benzenederived anchoring molecules, the catechol-based dye injects faster than the dye attached via a carboxylic acid group. This lower relative rate of carboxylic acid integrated in the dye can be explained by the change of adsorption mode: while benzoic acid stays in the bidentate mode, ben-NDI prefers monodental
Table 2. Slope, Adjusted $R^{2}$ Values and Estimated Electron Injection Rates for cat-NDI, ben-NDI and hyd-NDI Determined from the Linear Fits on the Semi Logarithmic Mean Value of 5 ETD Simulations Performed on Fully Solvated Nuclear Trajectories

\begin{tabular}{llccc} 
molecule & slope $\left[\mathrm{fs}^{-1}\right]$ & $\begin{array}{c}\text { adjusted } \\
R^{2}\end{array}$ & $\begin{array}{c}\text { estimated electron } \\
\text { injection rate }\left[\mathrm{s}^{-1}\right]\end{array}$ & $\begin{array}{c}\text { mean life } \\
\text { time }[\mathrm{fs}]\end{array}$ \\
cat-NDI & -0.028 & 0.96446 & $2.8 \times 10^{13}$ & 35 \\
ben-NDI & $-4.37 \times 10^{-3}$ & 0.99203 & $4.37 \times 10^{12}$ & 224 \\
hyd-NDI & $-2.21 \times 10^{-3}$ & 0.97753 & $2.21 \times 10^{12}$ & 452 \\
\hline
\end{tabular}

adsorption because of steric constraints, leading to a comparably lower injection rate.

\section{CONCLUSIONS}

We have investigated the photoinduced electron injection from three different anchoring molecules (catechol, benzoic acid, and benzohydroxamic acid) as well as from core-extended NDI-based dyes with these three anchoring moieties. An evaluation of the importance of nuclear dynamics, conformational averaging, and explicit solvation for the injection process was done. Nuclear dynamics are crucial for describing the electron injection process, suppressing oscillations back to the dye molecules and localizing the electron through the dynamic disorder. Conformational and trajectory averaging has a profound impact, especially in benzoic acid and hydroxamic acid derivatives. This is further underlined by the fact that the injection rate is strongly dependent on the anchoring mode (bidentate bridging $v s$ monodentate). Explicit solvation is of importance regarding the correct adsorption mode of anchoring groups and correct overall dynamics of the dyes. This holds especially true for hydroxamic acid. For the nuclear trajectory generation, explicit water should therefore be included. Explicit solvent polarization effects are low because of the tight binding character of the Hamiltonian. Concerning all anchor-dye complexes explored, cat-NDI emerged as the most effective (full injection within $150 \mathrm{fs}$ ). Ben-NDI (with $90 \%$ within $500 \mathrm{fs}$ ) and hyd-NDI (65\% within $500 \mathrm{fs}$ ) showed lower injection rates. The computational strategy employed, including the effect of nuclear dynamics, trajectory averaging, and explicit solvation at a still reasonable computational cost, allows for a more unbiased screening of potential molecular dyes and may be relevant in the development of new dyesensitized photoanodes for solar energy conversion.

\section{ASSOCIATED CONTENT}

SI Supporting Information

The Supporting Information is available free of charge at https://pubs.acs.org/doi/10.1021/acs.jpcc.0c09551.

Computational details, parameter optimization, influence of dihedral angle on electron injection, resonance effects in the injection process, and detailed graphs on photoinduced electron injection (PDF)

\section{AUTHOR INFORMATION}

\section{Corresponding Authors}

Jan Paul Menzel - Leiden Institute of Chemistry, Leiden University, 2300 RA Leiden, The Netherlands; (1) orcid.org/ 0000-0002-1312-5000; Email: j.p.menzel@

lic.leidenuniv.nl 
Francesco Buda - Leiden Institute of Chemistry, Leiden University, 2300 RA Leiden, The Netherlands; 10 orcid.org/ 0000-0002-7157-7654; Email: f.buda@chem.leidenuniv.nl

\section{Authors}

Anastasios Papadopoulos - Leiden Institute of Chemistry, Leiden University, 2300 RA Leiden, The Netherlands

Jelena Belic - Department of Chemistry and Pharmaceutical Sciences, Vrije Universiteit Amsterdam, Amsterdam 1081 $H V$, The Netherlands

Huub J. M. de Groot - Leiden Institute of Chemistry, Leiden University, 2300 RA Leiden, The Netherlands; 10 orcid.org/ 0000-0002-8796-1212

Lucas Visscher - Department of Chemistry and Pharmaceutical Sciences, Vrije Universiteit Amsterdam, Amsterdam $1081 \mathrm{HV}$, The Netherlands; 10 orcid.org/00000002-7748-6243

Complete contact information is available at: https://pubs.acs.org/10.1021/acs.jpcc.0c09551

\section{Author Contributions}

The manuscript was written through contributions of all authors. All authors have given approval to the final version of the manuscript.

Notes

The authors declare no competing financial interest.

\section{ACKNOWLEDGMENTS}

This research has been financially supported by the NWO Solar to Products program (project number 733.000.007). We acknowledge the use of supercomputer facilities at SURFsara sponsored by NWO Physical Sciences, with financial support from The Netherlands Organization for Scientific Research (NWO).

\section{REFERENCES}

(1) O’Regan, B.; Grätzel, M. A Low-Cost, High-Efficiency Solar Cell Based on Dye-Sensitized Colloidal TiO2 Films. Nature 1991, 353, 737-740.

(2) Cao, Y.; Saygili, Y.; Ummadisingu, A.; Teuscher, J.; Luo, J.; Pellet, N.; Giordano, F.; Zakeeruddin, S. M.; Moser, J.-E.; Freitag, M.; Hagfeldt, A.; Grätzel, M. 11\% Efficiency Solid-State Dye-Sensitized Solar Cells with Copper(II/I) Hole Transport Materials. Nat. Commun. 2017, 8, 15390.

(3) Zhang, L.; Yang, X.; Wang, W.; Gurzadyan, G. G.; Li, J.; Li, X.; An, J.; Yu, Z.; Wang, H.; Cai, B.; et al. 13.6\% Efficient Organic DyeSensitized Solar Cells by Minimizing Energy Losses of the Excited State. ACS Energy Lett. 2019, 4, 943-951.

(4) Hagfeldt, A.; Boschloo, G.; Sun, L.; Kloo, L.; Pettersson, H. DyeSensitized Solar Cells. Chem. Rev. 2010, 110, 6595-6663.

(5) Li, F.; Fan, K.; Xu, B.; Gabrielsson, E.; Daniel, Q.; Li, L.; Sun, L. Organic Dye-Sensitized Tandem Photoelectrochemical Cell for Light Driven Total Water Splitting. J. Am. Chem. Soc. 2015, 137, 91539159.

(6) Yamamoto, M.; Nishizawa, Y.; Chábera, P.; Li, F.; Pascher, T.; Sundström, V.; Sun, L.; Imahori, H. Visible Light-Driven Water Oxidation with a Subporphyrin Sensitizer and a Water Oxidation Catalyst. Chem. Commun. 2016, 52, 13702-13705.

(7) Jiang, J.; Spies, J. A.; Swierk, J. R.; Matula, A. J.; Regan, K. P.; Romano, N.; Brennan, B. J.; Crabtree, R. H.; Batista, V. S.; Schmuttenmaer, C. A.; Brudvig, G. W. Direct Interfacial Electron Transfer from High-Potential Porphyrins into Semiconductor Surfaces: A Comparison of Linkers and Anchoring Groups. J. Phys. Chem. C 2018, 122, 13529-13539.
(8) Massin, J.; Bräutigam, M.; Bold, S.; Wächtler, M.; Pavone, M.; Muñoz-García, A. B.; Dietzek, B.; Artero, V.; Chavarot-Kerlidou, M. Investigating Light-Driven Hole Injection and Hydrogen Evolution Catalysis at Dye-Sensitized NiO Photocathodes: A Combined Experimental-Theoretical Study. J. Phys. Chem. C 2019, 123, 17176-17184.

(9) Yu, Z.; Li, F.; Sun, L. Recent Advances in Dye-Sensitized Photoelectrochemical Cells for Solar Hydrogen Production Based on Molecular Components. Energy Environ. Sci. 2015, 8, 760-775.

(10) Haque, S. A.; Palomares, E.; Cho, B. M.; Green, A. N. M.; Hirata, N.; Klug, D. R.; Durrant, J. R. Charge Separation versus Recombination in Dye-Sensitized Nanocrystalline Solar Cells: The Minimization of Kinetic Redundancy. J. Am. Chem. Soc. 2005, 127, 3456-3462.

(11) Maggio, E.; Martsinovich, N.; Troisi, A. Evaluating Charge Recombination Rate in Dye-Sensitized Solar Cells from Electronic Structure Calculations. J. Phys. Chem. C 2012, 116, 7638-7649.

(12) Mai, S.; González, L. Molecular Photochemistry: Recent Developments in Theory. Angew. Chem., Int. Ed. 2020, 59, 1683216846.

(13) Persico, M.; Granucci, G. Photochemistry. A Modern Theoretical Perspective; Springer: Cham (Switzerland), 2018.

(14) Belić, J.; van Beek, B.; Menzel, J. P.; Buda, F.; Visscher, L. Systematic Computational Design and Optimization of Light Absorbing Dyes. J. Phys. Chem. A 2020, 124, 6380-6388.

(15) Le Bahers, T.; Labat, F.; Pauporté, T.; Lainé, P. P.; Ciofini, I. Theoretical Procedure for Optimizing Dye-Sensitized Solar Cells: From Electronic Structure to Photovoltaic Efficiency. J. Am. Chem. Soc. 2011, 133, 8005-8013.

(16) De Angelis, F.; Fantacci, S.; Selloni, A.; Grätzel, M.; Nazeeruddin, M. K. Influence of the Sensitizer Adsorption Mode on the Open-Circuit Potential of Dye-Sensitized Solar Cells. Nano Lett. 2007, 7, 3189-3195.

(17) Cheng, J.; Sulpizi, M.; VandeVondele, J.; Sprik, M. Hole Localization and Thermochemistry of Oxidative Dehydrogenation of Aqueous Rutile $\mathrm{TiO} 2(110)$. ChemCatChem 2012, 4, 636-640.

(18) Yang, L.; Lindblad, R.; Gabrielsson, E.; Boschloo, G.; Rensmo, H.; Sun, L.; Hagfeldt, A.; Edvinsson, T.; Johansson, E. M. J. Experimental and Theoretical Investigation of the Function of 4-TertButyl Pyridine for Interface Energy Level Adjustment in Efficient Solid-State Dye-Sensitized Solar Cells. ACS Appl. Mater. Interfaces 2018, 10, 11572-11579.

(19) Pastore, M.; Mosconi, E.; De Angelis, F.; Grätzel, M. A Computational Investigation of Organic Dyes for Dye-Sensitized Solar Cells: Benchmark, Strategies, and Open Issues. J. Phys. Chem. C 2010, 114, 7205-7212.

(20) Pastore, M.; Fantacci, S.; De Angelis, F. Ab Initio Determination of Ground and Excited State Oxidation Potentials of Organic Chromophores for Dye-Sensitized Solar Cells. J. Phys. Chem. C 2010, 114, 22742-22750.

(21) De Angelis, F.; Fantacci, S.; Gebauer, R. Simulating DyeSensitized TiO2 Heterointerfaces in Explicit Solvent: Absorption Spectra, Energy Levels, and Dye Desorption. J. Phys. Chem. Lett. 2011, 2, 813-817.

(22) Akimov, A. V.; Neukirch, A. J.; Prezhdo, O. V. Theoretical Insights into Photoinduced Charge Transfer and Catalysis at Oxide Interfaces. Chem. Rev. 2013, 113, 4496-4565.

(23) Curchod, B. F. E.; Rothlisberger, U.; Tavernelli, I. TrajectoryBased Nonadiabatic Dynamics with Time-Dependent Density Functional Theory. Chem. Phys. Chem. 2013, 14, 1314-1340.

(24) Rozzi, A. C.; Falke, M. S.; Spallanzani, N.; Rubio, A.; Molinari, E.; Brida, D.; Maiuri, M.; Cerullo, G.; Schramm, H.; Christoffers, J.; Lienau, C. Quantum Coherence Controls the Charge Separation in a Prototypical Artificial Light-Harvesting System. Nat. Commun. 2013, 4, 1602.

(25) Eisenmayer, T. J.; Buda, F. Real-Time Simulations of Photoinduced Coherent Charge Transfer and Proton-Coupled Electron Transfer. ChemPhysChem 2014, 15, 3258-3263. 
(26) Menzel, J. P.; de Groot, H. J. M.; Buda, F. Photoinduced Electron Transfer in Donor-Acceptor Complexes: Isotope Effect and Dynamic Symmetry Breaking. J. Phys. Chem. Lett. 2019, 10, 65046511.

(27) Meng, S.; Ren, J.; Kaxiras, E. Natural Dyes Adsorbed on $\mathrm{TiO}_{2}$ Nanowire for Photovoltaic Applications: Enhanced Light Absorption and Ultrafast Electron Injection. Nano Lett. 2008, 8, 3266-3272.

(28) Duncan, W. R.; Prezhdo, O. V. Temperature Independence of the Photoinduced Electron Injection in Dye-Sensitized $\mathrm{TiO} 2$ Rationalized by $\mathrm{Ab}$ Initio Time-Domain Density Functional Theory. J. Am. Chem. Soc. 2008, 130, 9756-9762.

(29) Meng, S.; Kaxiras, E. Electron and Hole Dynamics in DyeSensitized Solar Cells: Influencing Factors and Systematic Trends. Nano Lett. 2010, 10, 1238-1247.

(30) Kolesov, G.; GrÅnäs, O.; Hoyt, R.; Vinichenko, D.; Kaxiras, E. Real-Time TD-DFT with Classical Ion Dynamics: Methodology and Applications. J. Chem. Theory Comput. 2016, 12, 466-476.

(31) Long, R.; Casanova, D.; Fang, W.-H.; Prezhdo, O. V. DonorAcceptor Interaction Determines the Mechanism of Photoinduced Electron Injection from Graphene Quantum Dots into $\mathrm{TiO}_{2}: \pi$ Stacking Supersedes Covalent Bonding. J. Am. Chem. Soc. 2017, 139, 2619-2629.

(32) Rego, L. G. C.; Batista, V. S. Quantum Dynamics Simulations of Interfacial Electron Transfer in Sensitized $\mathrm{TiO}_{2}$ Semiconductors. J. Am. Chem. Soc. 2003, 125, 7989-7997.

(33) Rego, L. G. C.; Abuabara, S. G.; Batista, V. S. Coherent Optical Control of Electronic Excitations in Functionalized Semiconductor Nanostructures. Quantum Info. Comput. 2005, 5, 318-334.

(34) Rego, L. G. C.; Abuabara, S. G.; Batista, V. S. Model Study of Coherent Quantum Dynamics of Hole States in Functionalized Semiconductor Nanostructures. J. Chem. Phys. 2005, 122, 154709.

(35) Rego, L. G. C.; Abuabara, S. G.; Batista, V. S. Coherent Control of Tunnelling Dynamics in Functionalized Semiconductor Nanostructures: A Quantum-Control Scenario Based on Stochastic Unitary Pulses. J. Mod. Opt. 2006, 53, 2519-2532.

(36) Xiao, D.; Martini, L. A.; Snoeberger, R. C.; Crabtree, R. H.; Batista, V. S. Inverse Design and Synthesis of Acac-Coumarin Anchors for Robust TiO2 Sensitization. J. Am. Chem. Soc. 2011, 133, 90149022.

(37) Monti, A.; Negre, C. F. A.; Batista, V. S.; Rego, L. G. C.; de Groot, H. J. M.; Buda, F. Crucial Role of Nuclear Dynamics for Electron Injection in a Dye-Semiconductor Complex. J. Phys. Chem. Lett. 2015, 6, 2393-2398.

(38) Poddutoori, P. K.; Thomsen, J. M.; Milot, R. L.; Sheehan, S. W.; Negre, C. F. A.; Garapati, V. K. R.; Schmuttenmaer, C. A.; Batista, V. S.; Brudvig, G. W.; van der Est, A. Interfacial Electron Transfer in Photoanodes Based on Phosphorus(v) Porphyrin Sensitizers CoDeposited on $\mathrm{SnO}_{2}$ with the $\mathrm{Ir}(\mathrm{III}) \mathrm{Cp} *$ Water Oxidation Precatalyst. J. Mater. Chem. A 2015, 3, 3868-3879.

(39) McNamara, W. R.; Snoeberger, R. C., III; Li, G.; Richter, C.; Allen, L. J.; Milot, R. L.; Schmuttenmaer, C. A.; Crabtree, R. H.; Brudvig, G. W.; Batista, V. S. Hydroxamate Anchors for Water-Stable Attachment to TiO2 Nanoparticles. Energy Environ. Sci. 2009, 2, 1173.

(40) Lim, G. N.; Hedström, S.; Jung, K. A.; Smith, P. A. D.; Batista, V. S.; D'Souza, F.; van der Est, A.; Poddutoori, P. K. Interfacial Electron Transfer Followed by Photooxidation in N,N-Bis(pAnisole)Aminopyridine-Aluminum(III) Porphyrin-Titanium(IV) Oxide Self-Assembled Photoanodes. J. Phys. Chem. C 2017, 121, 14484-14497.

(41) Lee, S. H.; Regan, K. P.; Hedström, S.; Matula, A. J.; Chaudhuri, S.; Crabtree, R. H.; Batista, V. S.; Schmuttenmaer, C. A.; Brudvig, G. W. Linker Length-Dependent Electron-Injection Dynamics of Trimesitylporphyrins on SnO2 Films. J. Phys. Chem. C 2017, 121, 22690-22699.

(42) Tichnell, C. R.; Miller, J. N.; Liu, C.; Mukherjee, S.; Jakubikova, E.; McCusker, J. K. Influence of Electrolyte Composition on Ultrafast Interfacial Electron Transfer in Fe-Sensitized TiO2-Based Solar Cells. J. Phys. Chem. C 2020, 124, 1794-1811.
(43) Shahroosvand, H.; Abaspour, S.; Pashaei, B.; Bideh, B. N. On How Ancillary Ligand Substitution Affects the Charge Carrier Dynamics in Dye-Sensitized Solar Cells. RSC Adv. 2018, 8, 1946519469.

(44) Shahroosvand, H.; Abbasi, P.; Bideh, B. N. Dye-Sensitized Solar Cell Based on Novel Star-Shaped Ruthenium Polypyridyl Sensitizer: New Insight into the Relationship between Molecular Designing and Its Outstanding Charge Carrier Dynamics. ChemistrySelect 2018, 3, 6821-6829.

(45) Abuabara, S. G.; Cady, C. W.; Baxter, J. B.; Schmuttenmaer, C. A.; Crabtree, R. H.; Brudvig, G. W.; Batista, V. S. Ultrafast Photooxidation of $\mathrm{Mn}(\mathrm{II})-$ Terpyridine Complexes Covalently Attached to $\mathrm{TiO}{ }_{2}$ Nanoparticles. J. Phys. Chem. C 2007, 111, 11982-11990.

(46) Mukherjee, S.; Liu, C.; Jakubikova, E. Comparison of Interfacial Electron Transfer Efficiency in $[\mathrm{Fe}(\mathrm{Ctpy}) 2] 2+-\mathrm{TiO} 2$ and $[\mathrm{Fe}-$ (CCNC)2]2+-TiO2 Assemblies: Importance of Conformational Sampling. J. Phys. Chem. A 2018, 122, 1821-1830.

(47) McNamara, W. R.; Snoeberger, R. C.; Li, G.; Schleicher, J. M.; Cady, C. W.; Poyatos, M.; Schmuttenmaer, C. A.; Crabtree, R. H.; Brudvig, G. W.; Batista, V. S. Acetylacetonate Anchors for Robust Functionalization of $\mathrm{TiO}_{2}$ Nanoparticles with $\mathrm{Mn}(\mathrm{II})$-Terpyridine Complexes. J. Am. Chem. Soc. 2008, 130, 14329-14338.

(48) Negre, C. F. A.; Young, K. J.; Oviedo, M. B.; Allen, L. J.; Sánchez, C. G.; Jarzembska, K. N.; Benedict, J. B.; Crabtree, R. H.; Coppens, P.; Brudvig, G. W.; Batista, V. S. Photoelectrochemical Hole Injection Revealed in Polyoxotitanate Nanocrystals Functionalized with Organic Adsorbates. J. Am. Chem. Soc. 2014, 136, 16420-16429.

(49) Hoff, D. A.; Silva, R.; Rego, L. G. C. Subpicosecond Dynamics of Metal-to-Ligand Charge-Transfer Excited States in Solvated $[\mathrm{Ru}($ Bpy) 3$] 2+$ Complexes. J. Phys. Chem. C 2011, 115, 1561715626.

(50) da Silva, R.; Hoff, D. A.; Rego, L. G. C. Coupled QuantumClassical Method for Long Range Charge Transfer: Relevance of the Nuclear Motion to the Quantum Electron Dynamics. J. Phys.: Condens. Matter 2015, 27, 134206.

(51) Hoff, D. A.; da Silva, R.; Rego, L. G. C. Coupled ElectronHole Quantum Dynamics on D- $\pi-\mathrm{A}$ Dye-Sensitized $\mathrm{TiO}_{2}$ Semiconductors. J. Phys. Chem. C 2012, 116, 21169-21178.

(52) Torres, A.; Oliboni, R. S.; Rego, L. G. C. Vibronic and Coherent Effects on Interfacial Electron Transfer Dynamics. J. Phys. Chem. Lett. 2015, 6, 4927-4935.

(53) da Silva Oliboni, R.; Bortolini, G.; Torres, A.; Rego, L. G. C. A Nonadiabatic Excited State Molecular Mechanics/Extended Hückel Ehrenfest Method. J. Phys. Chem. C 2016, 120, 27688-27698.

(54) Torres, A.; Prado, L. R.; Bortolini, G.; Rego, L. G. C. Charge Transfer Driven Structural Relaxation in a Push-Pull Azobenzene Dye-Semiconductor Complex. J. Phys. Chem. Lett. 2018, 9, 59265933.

(55) Oliboni, R. S.; Yan, H.; Fan, H.; Abraham, B.; Avenoso, J. P.; Galoppini, E.; Batista, V. S.; Gundlach, L.; Rego, L. G. C. Vibronic Effects in the Ultrafast Interfacial Electron Transfer of PeryleneSensitized TiO2 Surfaces. J. Phys. Chem. C 2019, 123, 12599-12607.

(56) Ambrosio, F.; Martsinovich, N.; Troisi, A. What Is the Best Anchoring Group for a Dye in a Dye-Sensitized Solar Cell? J. Phys. Chem. Lett. 2012, 3, 1531-1535.

(57) Sakai, N.; Mareda, J.; Vauthey, E.; Matile, S. Core-Substituted Naphthalenediimides. Chem. Commun. 2010, 46, 4225-4237.

(58) Narsaria, A. K.; Ruijter, J. D.; Hamlin, T. A.; Ehlers, A. W.; Guerra, C. F.; Lammertsma, K.; Bickelhaupt, F. M. Performance of TDDFT Vertical Excitation Energies of Core-Substituted Naphthalene Diimides. J. Comput. Chem. 2020, 41, 1448-1455.

(59) Elstner, M.; Porezag, D.; Jungnickel, G.; Elsner, J.; Haugk, M.; Frauenheim, T.; Suhai, S.; Seifert, G. Self-Consistent-Charge DensityFunctional Tight-Binding Method for Simulations of Complex Materials Properties. Phys. Rev. B 1998, 58, 7260-7268.

(60) Elstner, M.; Frauenheim, T.; Kaxiras, E.; Seifert, G.; Suhai, S. A Self-Consistent Charge Density-Functional Based Tight-Binding 
Scheme for Large Biomolecules. Phys. Status Solidi B 2000, 217, 357376.

(61) Frauenheim, T.; Seifert, G.; Elsterner, M.; Hajnal, Z.; Jungnickel, G.; Porezag, D.; Suhai, S.; Scholz, R. A Self-Consistent Charge Density-Functional Based Tight-Binding Method for Predictive Materials Simulations in Physics, Chemistry and Biology. Phys. Status Solidi B 2000, 217, 41-62.

(62) ADF 2019.3, SCM, Theoretical Chemistry; Vrije Universiteit: Amsterdam, The Netherlands, http://www.scm.com.

(63) te Velde, G.; Bickelhaupt, F. M.; Baerends, E. J.; Fonseca Guerra, C.; van Gisbergen, S. J. A.; Snijders, J. G.; Ziegler, T. Chemistry with ADF. J. Comput. Chem. 2001, 22, 931-967.

(64) Dolgonos, G.; Aradi, B.; Moreira, N. H.; Frauenheim, T. An Improved Self-Consistent-Charge Density-Functional Tight-Binding (SCC-DFTB) Set of Parameters for Simulation of Bulk and Molecular Systems Involving Titanium. J. Chem. Theory Comput. 2010, 6, 266278.

(65) Luttrell, T.; Halpegamage, S.; Tao, J.; Kramer, A.; Sutter, E.; Batzill, M. Why Is Anatase a Better Photocatalyst than Rutile? - Model Studies on Epitaxial TiO 2 Films. Sci. Rep. 2014, 4, 4043.

(66) Rudshteyn, B.; Negre, C. F. A.; Oliboni, R. S.; Monti, A.; Chen, J.; Crabtree, R. H.; Rego, L. G. C.; Batista, V. S. Inferring Protonation States of Hydroxamate Adsorbates on $\mathrm{TiO}_{2}$ Surfaces. J. Phys. Chem. C 2017, 121, 11985-11990.

(67) Berendsen, H. J. C.; Postma, J. P. M.; van Gunsteren, W. F.; DiNola, A.; Haak, J. R. Molecular Dynamics with Coupling to an External Bath. J. Chem. Phys. 1984, 81, 3684-3690.

(68) Alvarez, S. Table of Parameters for Extended Huckel Calculations, Collected by Santiago Alvarez; Universitat de Barcelona, 1995; p 1995.

(69) Becke, A. D. New Mixing of Hartree-Fock and Local Densityfunctional Theories. J. Chem. Phys. 1993, 98, 1372-1377.

(70) Lee, C.; Yang, W.; Parr, R. G. Development of the ColleSalvetti Correlation-Energy Formula into a Functional of the Electron Density. Phys. Rev. B: Condens. Matter Mater. Phys. 1988, 37, 785789.

(71) Van Lenthe, E.; Baerends, E. J. Optimized Slater-Type Basis Sets for the Elements 1-118. J. Comput. Chem. 2003, 24, 1142-1156.

(72) Pye, C. C.; Ziegler, T. An Implementation of the Conductorlike Screening Model of Solvation within the Amsterdam Density Functional Package. Theor. Chem. Acc. 1999, 101, 396-408.

(73) Ooyama, Y.; Yamaji, K.; Ohshita, J. Photovoltaic Performances of Type-II Dye-Sensitized Solar Cells Based on Catechol Dye Sensitizers: Retardation of Back-Electron Transfer by PET (PhotoInduced Electron Transfer). Mater. Chem. Front. 2017, 1, 2243-2255.

(74) Selli, D.; Fazio, G.; Seifert, G.; Di Valentin, C. Water Multilayers on TiO2 (101) Anatase Surface: Assessment of a DFTBBased Method. J. Chem. Theory Comput. 2017, 13, 3862-3873.

(75) Aschauer, U. J.; Tilocca, A.; Selloni, A. Ab Initio Simulations of the Structure of Thin Water Layers on Defective Anatase TiO2(101) Surfaces. Int. J. Quantum Chem. 2015, 115, 1250-1257. 